\title{
Dwarf Galaxies in the Coma Cluster. II. Photometry and Analysis
}

\author{
Jeff Secker \\ Program in Astronomy, Washington State University, Pullman, WA 99164-3113 USA \\ secker@delta.math.wsu.edu \\ William E. Harris ${ }^{1}$ \\ Department of Physics and Astronomy, McMaster University, Hamilton, Ontario L8S 4M1 \\ Canada \\ harris@physics.mcmaster.ca \\ Julia D. Plummer ${ }^{2}$ \\ Program in Astronomy, Washington State University, Pullman, WA 99164-3113 USA
}

\begin{abstract}
We use the data set derived in our previous paper (Secker \& Harris 1997) to study the dwarf galaxy population in the central $\simeq 700 \operatorname{arcmin}^{2}$ of the Coma cluster, the majority of which are early-type dwarf elliptical (dE) galaxies. Analysis of the statistically-decontaminated dE galaxy sequence in the color-magnitude diagram reveals that the mean $\mathrm{dE}$ color at $R=18.0$ mag is $(B-R) \simeq 1.4 \mathrm{mag}$, but that a highly significant trend of color with magnitude exists $(\Delta(B-R) / \Delta R=-0.056 \pm 0.002 \mathrm{mag})$ in the sense that fainter dEs are bluer and thus presumably more metal-poor. The mean color of the faintest dEs in our sample is $(B-R) \simeq 1.15 \mathrm{mag}$, consistent with a color measurement of the diffuse intracluster light in the Coma core. This intracluster light could then have originated from the tidal disruption of faint $\mathrm{dEs}$ in the cluster core. The total galaxy luminosity function (LF) is well modeled as the sum of a log-normal distribution for the giant galaxies, and a Schechter function for the dE galaxies with a faint-end slope $\alpha=-1.41 \pm 0.05$. This value of $\alpha$ is consistent with those measured for the Virgo and Fornax clusters. The spatial distribution of the faint dE galaxies $(19.0<R \leq 22.5 \mathrm{mag})$ is well fit by a standard King model with a central surface density of $\Sigma_{0}=1.17 \mathrm{dEs} \operatorname{arcmin}^{-2}$ and a core radius $R_{\mathrm{c}}=22.15$
\end{abstract}

\footnotetext{
${ }^{1}$ Visiting Astronomer, Kitt Peak National Observatory, operated by AURA, Inc. under contract to the National Science Foundation.

${ }^{2}$ Current address: Department of Astronomy, University of Michigan, 830 Dennison, 501 East University Avenue, Ann Arbor, MI 48109-1090; plummer@astro.lsa.umich.edu
} 
$\operatorname{arcmin}\left(\simeq 0.46 h^{-1} \mathrm{Mpc}\right)$. This core is significantly larger than the $R_{\mathrm{c}}=13.71$ $\operatorname{arcmin}\left(\simeq 0.29 h^{-1} \mathrm{Mpc}\right)$ found for the cluster giants and the brighter dEs ( $R \leq 19.0 \mathrm{mag}$ ), again consistent with the idea that faint dEs in the dense core have been disrupted. Finally, we find that most dEs belong to the general Coma cluster potential rather than as satellites of individual giant galaxies: An analysis of the number counts around 10 cluster giants reveals that they each have on average $4 \pm 1 \mathrm{dE}$ companions within a projected radius of $13.9 h^{-1} \mathrm{kpc}$.

Subject headings: galaxies: clusters: individual (Coma) - galaxies: formation - galaxies: evolution - galaxies: luminosity function - galaxies: dwarf: elliptical

\section{INTRODUCTION}

Like their giant-galaxy counterparts, dwarf galaxies are divided into early- and latetypes. Late-type dwarfs are found predominately in the field or the less dense regions of galaxy clusters, and are dominated in number by the dwarf irregular (dIr) galaxies, with a small contribution from dwarf spirals (Schombert et al. 1995). By contrast, dwarf ellipticals (dEs) are found in large numbers within rather dense, populous cluster environments. In this paper, we present a study of the properties of the dwarfs in the Coma cluster core, which are almost entirely of the dE type.

The prototypical dE is a smooth, centrally concentrated, low surface brightness galaxy. The majority of more luminous $\mathrm{dE}$ galaxies are nucleated $(\mathrm{dE}, \mathrm{N})$, in which a central spike contains up to 20 percent of the total luminosity of the galaxy (van den Bergh 1986; Ferguson \& Binggeli 1994; Durrell 1997). These nuclei are most likely supermassive star clusters with typical radii $\lesssim 50 \mathrm{kpc}$ (hence unresolved at the distance of Coma with ground-based resolution), and their integrated colors are usually indistinguishable from the surrounding galaxy (Caldwell \& Bothun 1987). The faintest dE galaxies, which have a less than average central concentration, are often referred to as dwarf spheroidals (dSph); see Gallagher \& Wyse (1994). This distinction is simply one of total magnitude, since the characteristics of $\mathrm{dE}$ galaxies (see below) carry over to the fainter dSph galaxies. For about 25 dwarf members of the Virgo cluster, Sandage \& Binggeli (1984) introduced the dS0 class. These rare objects are intended to be analogous to the regular S0 galaxies, and show evidence for a two-component structure (i.e., a core plus flattened disk). The dividing lines among all these various subclasses are approximate and can even differ significantly between individual studies. In our analysis to follow, we make no effort to separate the sample of $\mathrm{dE}$ 
galaxies into their morphological groups, simply because our image resolution is insufficient to do so. Thus in this paper, we define $\mathrm{dE}$ galaxies empirically as low luminosity, low surface brightness galaxies in the range $-19.5 \lesssim M_{R} \lesssim-9.5 \mathrm{mag}$, with colors typically near $1.3 \lesssim(B-R) \lesssim 1.5 \mathrm{mag}$.

The great majority of $\mathrm{dE}$ galaxies are located in the dense environment of rich clusters of galaxies. At the present time, the formation of $\mathrm{dE}$ galaxies in rich clusters is not well understood (Ferguson \& Binggeli 1994; Moore et al. 1996; Secker 1996), and so we are unsure of their age and stellar composition. While most are thought to be composed of a metal-poor stellar population similar in age to globular clusters (Ferguson \& Binggeli 1994), some unknown fraction will most likely be similar to the Local Group dE and dSph galaxies, many of which show evidence for more than one episode of star formation (Caldwell \& Bothun 1987; Smecker-Hane et al. 1994; Sarajedini \& Layden 1995). What appears to be known is that $\mathrm{dEs}$ form a population which is fundamentally different from regular elliptical (E) galaxies (although see Jerjen \& Binggeli 1997):

(a) dE galaxies in general become less luminous with decreasing central surface brightness, contrary to the class of regular E galaxies for which the core radius and central surface brightness increases toward fainter magnitude (Kormendy 1977; Caldwell \& Bothun 1987; Ferguson \& Binggeli 1994). These features provide the basis for visual discrimination between $\mathrm{dE}$ and $\mathrm{E}$ galaxies near the same total magnitude: the dE galaxies are those with the lower surface brightness.

(b) dEs are characterized by smooth (as compared to dIr) surface brightness profiles, with the non-nucleated dEs being well fit by a single exponential (Ichikawa et al. 1986; Ferguson \& Binggeli 1994) or a modified-exponential profile (Cellone, Forte \& Geisler 1994; Jerjen \& Binggeli 1997). This differs from E galaxies, whose light distribution is well described by a de Vaucouleurs $R^{1 / 4}$ law. Thus in principle, the bright dE galaxies can be distinguished from faint E galaxies (at the same magnitude) on the basis of their surface brightness profiles.

(c) while color gradients are observed in some dE galaxies, there does not appear to be any preference for red or blue color gradients (Durrell 1997). This differs from the giant ellipticals, which generally become bluer (a metallicity effect) at larger radius.

(d) dEs appear to be dominated by extensive and extremely massive dark-matter halos. Measurements of the central stellar velocity dispersions indicate that $M / L \propto L^{0.2}$ for Es, but that $M / L \propto L^{-0.4}$ for dEs (Ferguson \& Binggeli 1994). Typical values seem to range from $M / L=4$ or $5 M_{\odot} / L_{\odot}$ for bright dEs in Virgo, up to $M / L \simeq 100$ for Draco, a dSph in the Local Group (Bender, Paquet \& Nieto 1991; Peterson \& Caldwell 1993). This observed 
trend in $M / L$ for dEs is consistent with the models of Dekel \& Silk (1986): for the case of dominant dark-matter halos, they predict a mass-to-light ratio which varies as $L^{-0.37}$. That is, the efficiency in which gas is converted into stars decreases with luminosity for $\mathrm{dE}$ galaxies, as the lower-mass dwarfs lose a larger fraction of their gas.

There have been three recent photometric studies of the small-galaxy population in Coma, each of which has a significant overlap with ours: (1) Thompson \& Gregory (1993; extending the study of Thompson \& Gregory 1980) is based upon photographic $m_{b}$ magnitudes and $(b-r)$ colors, complete to a limiting magnitude of $m_{b}=20.0$ mag (i.e., $R \simeq 18.6 \mathrm{mag}$ ). Their survey covers an area of approximately four square degrees, and provides a thorough analysis of many aspects of the Coma dwarf galaxy population. (2) Biviano et al. (1995) utilize a combination of redshifts and $(b-r)$ colors, together with photographic magnitudes, to obtain a sample of cluster members complete to a limit of $m_{b}=20.0 \mathrm{mag}$. With this data set they complete a statistical analysis of the galaxy luminosity function for an area of the cluster core $1260 \mathrm{arcmin}^{2}$, centered upon NGC 4878 and NGC 4889. (3) Bernstein et al. (1995) analyze deep $R$-band CCD images taken with the KPNO 4-meter telescope in $1.3^{\prime \prime}$ seeing. Their single field is $56 \mathrm{arcmin}^{2}$, located to the south of both NGC 4889 and NGC 4874. They perform number counts of all objects to a limit of $R=25.5 \mathrm{mag}$, with an excellent job of statistical number correction using five randomly-selected control fields.

The primary difference between these studies and ours is that we combine a moderately large spatial coverage $\left(\simeq 700 \operatorname{arcmin}^{2}\right)$ with accurate $R$ - and $B$-band CCD photometry and a moderately deep limiting magnitude. Our cluster membership is based upon the object's $(B-R)$ color, and as illustrated by Biviano et al. (1995; a comparison of redshift-selected and color-selected cluster definitions), this technique results in a minimal contamination from background galaxies. As described in our companion Paper I (Secker \& Harris 1997), our CCD images cover a large fraction of the core environment of the Coma cluster, i.e. the region surrounding the supergiants NGC 4874 and NGC 4889, with another field extending southward to 23 arcmin from NGC 4874. These images provide an extremely large sample of candidate dwarf galaxies, the vast majority of which are the early-type dEs, and our analysis of this sample yields significant correlations relevant to their formation and subsequent dynamical evolution in this ultradense environment.

In Paper I we describe the observations and data reduction techniques for a sample of candidate cluster galaxies. The dES are clearly evident in the color-magnitude diagram (CMD) as a tightly defined sequence of objects not present on the background control field. In this paper we analyze several features of our database for the $\mathrm{dE}$ galaxy population: In Section 2, we discuss sample definition, photometric calibration and magnitude 
completeness. In Section 3, we analyze the dependence of dE color on integrated magnitude, using the control-field subtracted color-magnitude diagram. In Section 4 we compute the faint-end slope of the Schechter function, and model the net galaxy luminosity function, decomposing it into two components. In Section 5, we analyze and compare control-field corrected radial number-density profiles for magnitude-selected subsamples of the cluster galaxies. Finally, in Section 6 we model and subtract a subset of the cluster giants, in an effort to quantify the number of bound companions in this dense environment.

\section{Sample Definition, Calibration and Completeness}

\subsection{Calibration Procedure}

The calibration of our $B$-and $R$-band magnitude zeropoints and other coefficients was accomplished with repeated observations of four standard star fields: M67 (Schild 1983), M92, NGC 2419 and NGC 4147 (Davis 1990), summarized in Paper I. To our $B$ - and $R$ standard star measurements we fit standard linear photometric transfer equations

$$
\begin{aligned}
R & =r-Z P_{R}-a_{1} X_{R}-a_{2}(B-R) \\
(B-R) & =\frac{\left[\left(b-Z P_{B}\right)-\left(r-Z P_{R}\right)\right]-a_{3} X_{B}+a_{1} X_{R}}{1-\left(a_{4}-a_{2}\right)}
\end{aligned}
$$

where $b$ and $r$ represent instrumental aperture magnitudes of the standard stars, $Z P_{B}$ and $Z P_{R}$ denote the magnitude zeropoint terms, $a_{1}$ and $a_{3}$ represent the extinction coefficients, and $a_{2}$ and $a_{4}$ represent the color coefficients. The $b$ and $r$ magnitudes were derived with DAOPHOT (Stetson 1987), by summing the pixel intensity within a 10-pixel aperture radius, subtracting the mode of the sky distribution computed within a sky annulus of inner radius 20 pixels and width of five pixels, and normalizing to a 1 second exposure.

We assume that the color coefficients are constant from night to night for a given

filter, whereas the zeropoint term and the airmass coefficient can vary between nights. For both nights, the coefficients of these transformation equations were determined using the simultaneous multilinear least squares reduction method developed by Harris et al. (1981). To best constrain our solutions, we adopted typical values for the extinction coefficients of $a_{1}=0.130$ and $a_{3}=0.283$ (Landolt 1982). Reducing both nights of data simultaneously (but $R$ and $B$ separately) yielded the zeropoints and coefficients given in Table 1. With the mean airmass values tabulated in Paper I, all quantities on the right-hand side of (11) are known, and our magnitudes and colors are completely calibrated. 


\subsection{Magnitude Completeness and Photometry Comparison}

The completeness of detection as a function of magnitude, $f(m)$, was first estimated by matching object detection lists from overlap regions for the three program-field $R$-band master images. There are two of these overlap regions: the North-South region, consisting of $280 \times 2000 \mathrm{px}^{2}$ in common between the NGC 4874 and NGC 4889 fields, and the East-West region, consisting of $1500 \times 450 \mathrm{px}^{2}$ in common between the NGC 4874 and the NGC 4874 South fields. These regions extend from the strong light gradients between NGC 4874 and NGC 4889 to the relatively flat sky levels south of NGC 4874 and thus provide a sensible overall average estimate for $f(m)$. As will be seen in more detail below, $f<0.8$ above our adopted limiting magnitude of $R=22.5 \mathrm{mag}$; thus there is less than a four percent chance that a real object will be missed on both images on an overlap region. An advantage to this method (as opposed to artificial star simulations; see Section 5) is that the objects we compare are real; they span a large range in surface brightness, and they have been through the complete range of image preprocessing, detection and measurement.

Consider first the East-West overlap region. We define $N_{T}(m)$ as the total (combined) number of objects detected on both the East and the West sections. Then, we define $N_{m}(m)$ as the number of matched objects; that is, the subset of objects detected on the West (NGC 4874) image, and detected (measured at any magnitude) on the corresponding East overlap region. For the purpose of binning, the object's "true" magnitude is adopted to be that measured on the NGC 4874 master image. Then the fractional completeness in each 0.5-magnitude bin is given by

$$
f(m)=\frac{N_{m}(m)}{N_{T}(m)-N_{m}(m)},
$$

and we adopt Bolte's (1989) expression for the uncertainty in $f(m)$, given by

$$
\sigma(f)=\frac{f(1-f)}{N_{T}}
$$

Results of this matching procedure from the two separate overlap regions were combined together to yield an averaged completeness function, for which the effects of small-number statistics are somewhat reduced.

In Figure 11 we plot $f(m)$ over the magnitude range of interest, derived using (2) and (通). The open circles denote the completeness function derived using all measured objects, while the solid circles denote the completeness function derived using the subsample of objects within the color range $0.7 \leq(B-R) \leq 1.9$ mag (Section 2.3). While these two 
functions are consistent within their uncertainty ranges, the color-restricted subsample is consistently more complete at all magnitudes. It is the $f(m)$ defined by this color-restricted sample which we adopt for statistical corrections in Section 4. Based upon an inspection of this completeness plot, and the behavior of the photometric error with magnitude (Paper I), we adopt a limiting magnitude of $R=22.5 \mathrm{mag}$, and discard all objects with total magnitudes below this limit. Note that the object sample used to define these functions was truncated at $R=23.5$. Since the scatter in the total magnitude is about 0.1 -mag at $R=22.5 \mathrm{mag}$, the faint end of our derived $f(m)$ is not affected by this truncation.

In the range $18 \leq R \leq 22.5 \mathrm{mag}$, the color restricted subsample of objects is well represented by a straight line, as plotted in Figure 1 (dashed line):

$$
f(m)=\left\{\begin{array}{rr}
-R / 22.5+1.8 ; & 18 \leq R \leq 22.5 \mathrm{mag} \\
1.0 ; & R<18.0 \mathrm{mag}
\end{array}\right.
$$

In addition to the magnitude incompleteness, incompleteness can also occur in color and spatial distributions. Brighter than $R=22.5 \mathrm{mag}$, we were able to measure $B$-band magnitudes for virtually all detected objects. (We discarded a handful of unmeasured objects, but well less than one percent.) Thus, we are essentially 100 percent complete for the color measurements. Concerning spatial incompleteness, we discard a circular region of radius 150 pixels centered on both of the two supergiant galaxies, and a circular region of radius 100 pixels centered on the bright saturated star located north of NGC 4874 . On the original master images, the light gradients in these regions were too extreme to permit adequate filtering and useful photometry. To a lesser extent, all of the giant (bright and extended) cluster galaxies contribute to a small (but non-negligible) degree of spatial incompleteness, as we cannot measure a small, faint, superimposed object using aperture photometry (Secker \& Harris 1996).

The set of all objects detected on both (i) an overlap region of the NGC 4874 image and on (ii) another of the program fields, can be used to compare total magnitudes and colors as a function of apparent total $R$ magnitude. In the top panel of Figure 2 we plot the magnitude difference $\Delta R$ mag versus the total magnitude, ranging from our completeness limit up to $R=14.5 \mathrm{mag}$. On this figure, $\Delta R$ is defined as the NGC 4874 magnitude minus the other measured magnitude; the cross symbols denote the 416 objects matched with the NGC 4889 field, and the solid circles denote the 448 objects matched with the NGC 4874 South field. It is immediately obvious that our magnitude scale is consistent between fields: the vast majority of the objects have $\Delta R \lesssim 0.10 \mathrm{mag}$, while those near the completeness limit have $\Delta R \lesssim 0.20 \mathrm{mag}$. However, a small bias exists, in the sense that the magnitudes measured on the NGC 4889 field are about 0.05 -mag brighter at all 
magnitudes. If this effect exists for the other overlap region, it is at a much lower level, and only occurs for the brighter objects. This discrepancy is most likely due to flat fielding errors, and while not completely negligible, these differences are sufficiently small that we do not attempt to correct for them. In the lower panel of Figure 2 we plot the color difference $\Delta(B-R)$ mag versus the total $R$ magnitude, for the same objects and the same magnitude range of the previous figure. It is evident that at all magnitudes, the agreement between the color scales is excellent, with no evidence for bias between fields.

\subsection{Combined Photometry Lists}

The calibrated object photometry lists for the four CCD fields described in Paper I (the three program fields NGC 4874, NGC 4889, NGC 4874 South and the Control field) were further culled to reduce contamination, according to the following restrictions. We discarded objects located within the bright stellar sequence defined by $r_{-2}<1.6$ pixels and $R<19.5 \mathrm{mag}$. (The pixel scale is $0.53 \mathrm{arcsec} / \mathrm{pixel}$, thus $1 \mathrm{px}=0.185 h^{-1} \mathrm{kpc}$.) The number of starlike objects in this region are 63, 59, 67 and 64 for the fields as listed above, consistent within Poisson statistics, as expected. The fractional contribution of galaxies

within this bright stellar sequence is negligible (see Paper I). However, for $R>19.5$ mag the starlike sequence is also populated with faint compact galaxies, which are more numerous on the program fields. To discard the sequence would impose an extreme bias against these compact objects. Thus in this region we depend upon statistical correction using the Control field to account for the contamination by starlike objects. Next, the point-like objects with $r_{-2} \lesssim 1.25$ pixels, and all objects with total magnitude below $R=22.5 \mathrm{mag}$ (our completeness limit), were culled from the sample. Then, all objects which overlapped the physical boundary of the CCD image were culled from the list, as were objects which overlapped another to such a degree that photometry was not possible (two to four percent of the original sample).

Finally, the calibrated object photometry lists for the three cluster fields were combined to obtain one master list. Since the three program fields share a considerable overlapping area, we culled multiple object detections in the following manner. For the North-South overlap region, the final object photometry is taken from the East edge of the NGC 4874 field, while for the East-West overlap region, the object photometry is taken from the North edge of the NGC 4874 South field. Note that we did not merge the object lists in the overlap regions, as this would artificially inflate the detection completeness in these regions. Two further notes are relevant. In the analysis of this paper, we use the observed $(B-R)$ colors, since reddening and extinction are negligible towards the North Galactic Pole. As 
discussed in Paper I, our $2 r_{1}$ aperture magnitudes are optimized for exponential-profile galaxies, and therefore they underestimate the total magnitudes for the giant ellipticals. The total magnitudes and colors we compute for the 70 brightest cluster galaxies are as accurate as possible given our analysis method, and we use these values for our analysis in this paper. However, other values in the literature may be more accurate, provided these objects were considered individually using isophote-fitting techniques.

Over the entire range in $(B-R)$ color and above $R=22.5 \mathrm{mag}$, there are 3723 objects in the final sample of program field objects, versus 1146 objects on the control field. For the analysis of Sections 3 through 6, we define a color-restricted subsample which includes objects with colors in the range $0.7 \leq(B-R) \leq 1.9$ mag. These extremes represent generous limits to the cluster early-type galaxy (dwarfs and giants) sequence, and reduce contamination from noncluster galaxies. This sample includes 2526 program-field objects and 694 Control field objects. The total area of the Coma cluster core covered by our program fields, minus the area of the overlap regions and the discarded regions around the cores of the two supergiant galaxies, is $A_{p}=698.44 \mathrm{arcmin}^{2}$. The total area of the Control field is $A_{c}=271.13 \mathrm{arcmin}^{2}$, thus $A_{p} / A_{c}=2.5760$. Then the number density of objects on the CONTROL field (integrated over all magnitudes) is given by $\overline{N_{c}}=2.560 \pm 0.097 \operatorname{arcmin}^{-2}$ for the color-restricted sample. Typical uncertainties at the $R=22.5$ limiting magnitude are $\sigma(R) \lesssim \pm 0.06 \mathrm{mag}$, and $\sigma(B-R) \lesssim \pm 0.12 \mathrm{mag}$. The photometry tables for the full sample of 3723 program field objects and 1146 control field objects are available electronically from the first author.

In Section 4, a luminosity function defined by the color-restricted ConTroL-field sample is used to correct the raw luminosity function observed on the program field. In addition, we use the integrated spatial number density to statistically correct the surface density profiles for our cluster dE galaxies in Section 5. We do this with the knowledge that it is only a first-order correction for the effect of uniformly distributed foreground stars and noncluster galaxies. A single control field provides an accurate estimate (to within Poisson uncertainties) of the number of genuine Galactic foreground stars, together with their luminosity and color distributions. However, a larger field-to-field variance arises in the number counts of faint galaxies since they reside predominantly in clusters and superclusters with apparent angular diameters on the order of several arcminutes. Thus the field-to-field variance in the observed number counts will definitely be larger than the Poisson $\sqrt{N}$ uncertainties. An average of several randomly offset control fields would give a more accurate mean number density and background luminosity function (e.g., Bernstein et al. 1995). In our analysis, we instead used an object's color to discriminate between cluster and non-cluster galaxies. As illustrated by Biviano et al. (1995), determination of cluster membership in this manner is comparable in effectiveness to redshift selection. 
In this manner we reduce the field-to-field variance to a level very close to the irreducible $\sqrt{(} N)$ amounts. For a comparison of control-field number densities with previous studies, refer to Secker \& Harris (1996).

\subsection{Preliminary Analysis of the Final Sample}

In this section we illustrate the properties of the color-restricted sample of candidate cluster galaxies defined above, visually, and in parameter spaces involving the total $R$ magnitude, the $(B-R)$ color, the intensity-weighted radial moment $r_{1}$, and the central surface brightness $I_{\mathrm{c}}$. For purposes of illustration, we show here grey-scale plots of two small areas $\left(512 \times 512 \mathrm{px}^{2}\right)$ of the original $R$-band master CCD images: Figure $3(\mathrm{a})$ is an area northeast of NGC 4889, and Figure 3(b) is south of NGC 4874. Here, the numerous dE galaxies are very evident as the centrally-concentrated low-surface-brightness objects, from moderately bright objects down to the faintest smudges available to the eye. To provide some guidance in separating bright dEs from faint Es, the brightest few dEs on each image are labeled.

In Figure @, we plot color-magnitude diagrams for the full sample of 3723 program-field objects and for the 1146 ConTROL-field objects. On the program-field CMD, the dE galaxy sequence is densely populated and it completely dominates the region restricted to $0.7 \leq(B-R) \leq 1.9 \mathrm{mag}$. This galaxy sequence is completely absent from the Control-field CMD, as expected. In Figure 5, we plot the radial moment $r_{1}$ (pixels) against the total $R$ magnitude for the color-restricted sample of $2526 \mathrm{E}$ and $\mathrm{dE}$ galaxy candidates and for the 694 ConTroL-field objects. The large excess of cluster galaxies, over and above the control field population, is evident. The most extended dE galaxies measured have $5 \lesssim r_{1} \lesssim 11$ pixels: for a pixel scale of $0.185 h^{-1} \mathrm{kpc} /$ pixel, and with $r_{1}=2 r_{0}$ for exponential profile objects, this corresponds to an exponential-disk scale radius of $0.46 h^{-1} \lesssim r_{0} \lesssim 1.02 h^{-1} \mathrm{kpc}$. The faintest $d E$ galaxies which we can resolve are at $R=21$ mag: here, $2 \lesssim r_{1} \lesssim 3.25$ pixels, which corresponds to $0.19 h^{-1} \lesssim r_{0} \lesssim 0.30 h^{-1} \mathrm{kpc}$. These values are consistent with the study of Bernstein et al. (1995), who measure scale radii in the range $250 \lesssim r_{0} \lesssim 450$ pc for $\mathrm{dE}$ galaxies in the range $22.5 \leq R \leq 23.0 \mathrm{mag}$.

In Figure 6 we plot the central surface brightness, $I_{\mathrm{c}}$, versus the total $R$ magnitude for the color-restricted sample of program and control field objects. The high- $I_{\mathrm{c}}$ stellar sequence can be seen to begin at $R=19.5 \mathrm{mag}$. Between $15.5 \leq R \leq 19.5 \mathrm{mag}$, the diffuse sequence of objects is dominated by cluster dE galaxies. Below $R \simeq 20 \mathrm{mag}$, as we lose our ability to discriminate between different profiles, the ensemble of objects merges with the stellar sequence. Our choice of a limiting magnitude is evident at $R=22.5$ mag; while we 
are complete in magnitude above this limit, in surface brightness we are complete to $I_{\mathrm{c}} \simeq 25$ $\mathrm{mag} / \operatorname{arcsec}^{2}$. Note, however, that we detected objects with $I_{\mathrm{c}} \simeq 26.5 \mathrm{mag} / \mathrm{arcsec}^{2}$, so as not to bias our sample against low surface brightness dE galaxies.

\section{The $R$ versus $(B-R)$ Color-magnitude Distribution}

An analysis of the photometric colors of dwarf (and other) galaxies can provide valuable information concerning stellar populations of individual galaxies and populations of galaxies (Caldwell \& Bothun 1987; Evans, Davies \& Phillipps, 1990; Garilli et al. 1992; Cellone, Forte \& Geisler 1994). In Secker and Harris (1996), the $(B-R)$ colors were used primarily as a discriminator to eliminate contamination due to non-cluster galaxies and foreground stars. However, the $(B-R)$ color index, like $(B-I)$ and the Washington $\left(C-T_{1}\right)$ color, is also a sensitive and accurate estimator of the total heavy element abundance (metallicity) for old stellar populations (Geisler \& Forte 1990; Couture, Harris \& Allwright 1991, 1992; Held \& Mould 1994; Secker 1996), and an analysis of the CMD is warranted. Note that while both age and metallicity affect absorption features in the spectra (and therefore the integrated color) of stellar populations, the effect of metal abundance dominates over age effects in the observed $(B-R)$ color of old populations such as dE galaxies (Worthey 1994). Thus in our sample of galaxies, we assume that the redder galaxies are, on a relative scale, more metal rich than the bluer galaxies (Secker 1996).

Figure 4 illustrates the color restriction (i.e., $0.7 \leq(B-R) \leq 1.9 \mathrm{mag}$ ) which represents generous limits on the early-type galaxy sequence: the most probable members of the Coma cluster are included, while the large contribution from red background galaxies is excluded. It is this data sample which we analyze here, and in the right panel of Figure 7, we reproduce this subset of the total cluster field CMD. The dE galaxy sequence begins at $R \simeq 15.5 \mathrm{mag}$, with a mean color of $(B-R) \simeq 1.54 \mathrm{mag}$. This galaxy sequence shows a strong trend for fainter dE galaxies to have (on average) a bluer color. This trend was also observed for the brighter dEs in Coma by Thompson \& Gregory (1993) and Biviano et al. (1995). To quantify this trend, we calculate the trimmed median $(B-R)$ color (the solid circles) in one-magnitude bins over the entire luminosity range. The upper portion of the galaxy sequence is well defined and has very little apparent contamination. Considering only this bright part of the galaxy sequence (i.e., $14.0<R<18.5 \mathrm{mag}$ ), we perform a least-squares fit of a straight line to the median color values. The resulting best-fit regression line is plotted (solid line) over the valid range in the left panel of Figure 7 , and it clearly provides an excellent fit to the data. This regression line is given by 


$$
(B-R)=(-0.056 \pm 0.002) R+(2.41 \pm 0.04) .
$$

Also plotted in the left panel of Figure 7 are open circles, which correspond to the mean color of control-field objects, calculated in the same 1 magnitude bins (but only below $R=18.5 \mathrm{mag}$ ). The dashed line is an extension of the upper line; it is not a fit to any of the fainter data points. The control-field sample is dominated by faint noncluster galaxies, and this is apparent here; the mean colors given by the open circles are in all cases redder then the extrapolated $\mathrm{dE}$ galaxy sequence (dashed line). At magnitudes fainter than $R=18.5 \mathrm{mag}$, the $\mathrm{dE}$ sequence spreads due to photometric error and it merges with the multitude of noncluster galaxies, which are clearly the dominant population (in number fraction) at these magnitudes. The effect is that the program-field mean color values (solid circles) deviate from the dashed line, redward, towards the control-field mean color values. Even using a trimmed median which is by definition less sensitive to outliers cannot yield the true mean colors for the faint end of the sparse dE galaxy sequence, as it is the dEs which are the outliers of a color distribution function dominated in number by non-cluster galaxies. Below, the question we address is whether this blue trend observed for the brighter $\mathrm{dE}$ galaxies is continuous and linear over the full magnitude range, down to our limit at $R=22.5$ mag. We determine that it is, and that the apparent flattening observed is caused by the redder noncluster galaxies which compose the majority of our program field sample at these faint magnitudes.

We adopt a method which is similar to Secker et al. (1995) and Kuhn et al. (1996), to derive a decontaminated version of the program-field CMD. Our procedure first creates binned versions of the program-field and the control fields CMDs, preserving the two-dimensional nature of the data, something which is impossible using a scaled and subtracted histogram. Once the CMDs are binned, the number counts in the control-field CMD are scaled by the ratio of areas, $A_{p} / A_{c}=2.5760$ (Section 2.3), and subtracted from the program-field CMD. In this manner we correct for a localized excess of control-field objects in the CMD of the program fields. We define $P(c, m)$ and $C(c, m)$ to represent the number of objects in the program-field and control-field CMDs respectively, computed in bins of height 1.0-mag in total $R$ magnitude and width of 0.1 magnitudes in $(B-R)$ color. Then the number of objects in each bin of the control-field subtracted CMD $N(c, m)$ is given by

$$
N(c, m)=P(c, m)-\frac{A_{p}}{A_{c}} C(c, m),
$$

where $N(c, m)$ is computed over the range $12.5 \geq R \geq 22.5$ mag and in the color range 
$0.7 \geq(B-R) \geq 1.9$ mag. The uncertainty on $N(c, m)$ is given by the $\sqrt{N_{i j}}$ counting uncertainties for the program- and control-field number counts added in quadrature.

In the right panel of Figure 7, we plot the binned, scaled and subtracted CMD, $N(c, m)$, corresponding to what we expect for Coma cluster members. There is one vertical line segment for each $N(c, m)$ bin, with a height proportional to the number of objects in that bin. In this case, the solid circles represent the mean corrected color, calculated for each 1-mag bin by averaging over all color bins, weighted by the number of objects in each bin. The associated errorbars are not intended to convey accurate uncertainty estimates; they simply represent the spread of objects about the median color (i.e., the semi-interquartile range) in the initial program-field CMD. Reproduced in the right panel is the solid regression line form the left panel, together with the extrapolated dotted line. That is, we did not perform a separate least-squares fit to the new mean color values. The important result here is that this regression line provides an excellent fit to the corrected mean color values for the dE galaxy sequence, over the full range $15.5 \leq R \leq 22.5$ mag. Any apparent reddening in the left panel can therefore be attributed entirely to contamination of the cluster sample by noncluster galaxies and foreground stars. Note that redward of the dE galaxy sequence and fainter than $R=19.0 \mathrm{mag}$, the number of cluster galaxies is consistent with zero, though there is considerable scatter, including a number of negative values for $N(c, m)$. This occurs because the control-field CMD provides only an estimate for the number and distribution of objects in color and magnitude expected on the program field, and it will not (and should not) subtract out perfectly.

The color-magnitude trend we observe in the Coma dEs is quite certainly a continuation of the sequence defined by the early-type cluster giants, and it is very similar to that observed recently in Fornax (but see Evans, Davies \& Phillipps 1990 for a different result). Cellone, Forte \& Geisler (1994) plot a $T_{1},\left(C-T_{1}\right)$ CMD (Washington filters) for a sample of 14 Fornax dEs and notice this color-magnitude correlation. From a linear fit to their tabulated data points, we estimate a decrease of $0.078 \pm 0.029 \mathrm{mag}$ in $\left(C-T_{1}\right)$ for each magnitude decrease in total $T_{1}$ magnitude. As stated earlier, we assume that integrated $(B-R)$ colors measured for our sample of dEs reflect metallicities of the underlying stellar populations, in the sense that redder dEs are more metal rich than bluer dEs. This is supported by Cellone, Forte \& Geisler (1994), who find that the metallicity-insensitive color index $\left(M-T_{1}\right)$ is essentially constant with magnitude (within the scatter) for the same sample of $14 \mathrm{dEs}$ in Fornax. For Fornax Es, Caldwell \& Bothun (1987) find an decrease of $0.08 \pm 0.012 \mathrm{mag}$ in $(U-V)$ for each magnitude decrease in total $B$ magnitude. This color-magnitude trend may in fact extend fainter to their sample of 11 $\mathrm{dEs}$, but with a much larger scatter. In order to compare the color-magnitude relationship for our Coma cluster dEs with those in Fornax, we adopt color-metallicity relationships 
derived from metallicities and integrated colors for Galactic globular clusters (from Harris 1996). These are given by $[\mathrm{Fe} / \mathrm{H}]=(3.44 \pm 0.09)(B-R)_{0}-(5.35 \pm 0.10)$ and $[\mathrm{Fe} / \mathrm{H}]$ $=(1.92 \pm 0.05)(U-V)_{0}-(2.97 \pm 0.04)$, and are valid over the range $-2.4 \lesssim[\mathrm{Fe} / \mathrm{H}]$ $\lesssim-0.2$. From Geisler \& Forte (1990), we adopt the color-metallicity relationship [Fe/H] $=2.35\left(C-T_{1}\right)_{0}-4.39$, which is also calibrated against Galactic globular clusters over a similar metallicity range. For our sample of Coma dEs, we therefore obtain a metallicity change with magnitude of $\Delta[\mathrm{Fe} / \mathrm{H}] / \Delta R=-0.19 \pm 0.01$ dex. For the Fornax dEs, we calculate $\Delta[\mathrm{Fe} / \mathrm{H}] / \Delta T_{1}=-0.18 \pm 0.07$ dex for the Cellone et al. (1994) sample, and estimate $\Delta[\mathrm{Fe} / \mathrm{H}] / \Delta B=-0.16 \pm 0.07$ dex for the Caldwell \& Bothun (1987) sample. These slopes are formally consistent, suggesting that a very similar phenomenon has been at work in both these cluster environments.

These color-luminosity correlations have a simple interpretation within the context of dE galaxy formation. As described by Dekel \& Silk (1986), the brighter (and more massive) $\mathrm{dE}$ galaxies have deeper gravitational potential wells, and therefore better able to retain the interstellar gas which became super heated and metal enriched during initial stages of the star-formation epoch. Although star formation in early-type galaxies is believed to be short lived (i.e., $\lesssim 10^{9}$ years), this is sufficient time for several generations of massive stars to form out of this metal-enriched gas, leading to an observed stellar population with is enhanced in metals (redder) compared to the fainter dE galaxies.

In Figure 7, the spread in the dE sequence, about the fiducial line and at any magnitude, exceeds that which can be attributed to photometric error. For the restricted (bright-end) range $15.5 \leq R \leq 18.0 \mathrm{mag}$, the total width is nearly constant at $\sigma_{\mathrm{obs}}=0.07$ mag. And from Paper 1, the typical photometric error in the color estimate for our sample of objects is $\sigma_{\text {err }} \lesssim 0.025 \mathrm{mag}$. We conclude that the intrinsic width in the color of $\mathrm{dE}$ galaxies (at bright magnitudes) is $\sigma_{\text {int }}=\left(\sigma_{\text {obs }}^{2}-\sigma_{\text {err }}^{2}\right)^{1 / 2} \simeq 0.065$ mag. This intrinsic scatter in the $\mathrm{dE}$ galaxy metallicity could reflect fragment-to-fragment differences in an early epoch of pre-enrichment, or local variations in the density of the intergalactic gas pressure (Babul \& Rees 1992; Secker 1996).

The diffuse intergalactic component of the Coma cluster light is estimated to be on the order of 25 - 30 percent of the total light (Thuan \& Kormendy 1977; Melnick, White \& Hoessel 1977; see also Uson et al. 1991 for a more recent discussion of the difficulties inherent in measuring this intracluster light). Thuan \& Kormendy (1977) report that for Coma, the diffuse light appears to be bluer than the light of the giant galaxies, at least within 14 arcminutes of the core. This is supported by Mattila (1977), whose photoelectric photometer measurements yield $(B-V)=0.54 \pm 0.18 \mathrm{mag}$, in an area free of galaxies. Though quite blue, the Mattila (1977) measurement is consistent with the most 
metal-poor old stellar populations such as globular clusters, which are in the range range $0.55 \lesssim(B-V) \lesssim 0.85 \mathrm{mag}$,

One possible origin of this low surface brightness diffuse light is stars tidally stripped from the outer regions of their parent galaxies. As illustrated in Figure 7 , the faint dE galaxies are the most metal poor (bluest) galaxies in this environment, and as discussed in Section 5, they are also the most easily disrupted, and we have evidence that a significant population of these $\mathrm{dE}$ galaxies have been tidally destroyed in the cluster core. Therefore, it is interesting to compare the color of this intracluster light with the median color for faint $\mathrm{dE}$ galaxies. From Figure 0, an approximate median color for the dE galaxies (at $R=22.5 \mathrm{mag})$ is $(B-R) \simeq 1.15 \mathrm{mag}$. To convert this to an equivalent $(B-V)$ color, we use the $(B-V)-(B-R)$ color-color relation for individual halo giants of Durrell, Harris $\&$ Pritchet (1994). If we assume this is approximately correct for the integrated colors of $\mathrm{dE}$ galaxies, our $(B-R) \mathrm{dE}$ color corresponds to $(B-V) \simeq 0.70 \mathrm{mag}$, which is within the color range found by Mattila (1977) and similar to an intermediate-metallicity globular cluster $([\mathrm{Fe} / \mathrm{H}] \simeq-1)$. Thus, our CMD analysis is consistent with a major component of this diffuse intergalactic light originating as stars tidally stripped from numerous faint $\mathrm{dE}$ galaxies. If so, the mean color of this diffuse light should be consistent with that of the faint $\mathrm{dE}$ galaxies at all clustercentric radii. A more accurate measurement of the diffuse light would be of great interest.

\section{The Galaxy Luminosity Function}

The probability distribution function of luminosity for galaxies in a cluster is a fundamental observable quantity which places physical constraints on galaxy formation and subsequent dynamical evolution. From a CDM, self-similar, stochastic model for galaxy formation (Press \& Schechter 1974), Schechter (1976) derived a robust analytical form to parameterize the luminosity function (LF),

$$
\Phi(L) d L=\phi_{\star}\left(\frac{L}{L_{\star}}\right)^{\alpha} \exp \left(-\frac{L}{L_{\star}}\right) \frac{d L}{L_{\star}} .
$$

Here $L_{\star}$ is a characteristic luminosity at which the slope of the Schechter function changes fairly abruptly, $\alpha$ is the slope of the power law for $L \ll L_{\star}$, and $\phi_{\star}$ provides the normalization (number per unit volume). For a given ensemble of galaxies, the Schechter LF provides a convenient model for the comparison of galaxy populations in differing environments. Recent observations strongly dictate that there is no universal LF: not only does it differ between field and cluster environments, there is significant observed 
variation between different cluster environments. While it appears that individual galaxy morphological classes have unique LFs, their relative contribution to the composite LF is a product of environment (Binggeli, Sandage \& Tammann, 1988). Although the Schechter function does not adequately describe the composite galaxy LF (giants plus dwarfs) for the Coma cluster, it does provide a good fit to the dE luminosity function (Sandage, Binggeli \& Tammann 1985; Thompson \& Gregory 1993; Biviano et al. 1995; Secker \& Harris 1996).

The deepest study of the LF for the Coma cluster core is that by Bernstein et al. (1995). Over the range in magnitude of $15.5 \leq R \leq 23.5 \mathrm{mag}$, they determine that the slope of the faint-end power law is $\alpha=-1.42 \pm 0.05$, with a constant faint-end slope over this entire magnitude range. As well, Thompson \& Gregory (1993) find that $\alpha \simeq-1.43$ for the faint-end of the LF in Coma. These values are consistent with findings for both the Virgo and Fornax clusters (Binggeli, Sandage \& Tammann 1985; Sandage, Binggeli \& Tammann 1985; Ferguson \& Sandage 1988). However, the LFs measured in the cores of other rich galaxy clusters (not including Coma) have been found to be much steeper at their limiting magnitudes: De Propris et al. (1995) find $\alpha \simeq-2$ for A2052, A2107, A2199 and A2666 $(z=0.035,0.042,0.031,0.026)$, Driver et al. (1994) find $\alpha \simeq-1.8$ for A963 $(z=0.21)$, and Wilson et al. (1997) find $\alpha \simeq-2$ for A665 and A1689 ( $z=0.18)$. Here, we determine the slope for the faint-end of the LF as defined by our sample of dE galaxies in the cluster core, and compare this to the findings cited above.

We consider the color-restricted subsample of Section 2, which consists of 2526 program-field objects (some fraction of which are $\mathrm{E}$ and $\mathrm{dE}$ galaxies in the cluster), and 694 control-field objects; objects in both samples satisfying $R \leq 22.5 \mathrm{mag}$ and $0.7 \leq(B-R) \leq 1.9 \mathrm{mag}$. As is commonly done, we plot the galaxy LF as the number of galaxies per unit magnitude. A change of variables involving the definition of magnitude, its derivative and an expression for the luminosity, yields

$$
\Phi(m)=\kappa\left\{10^{0.4\left(m_{\star}-m\right)}\right\}^{(\alpha+1)}\left[\exp \left(-10^{0.4\left(m_{\star}-m\right)}\right)\right]
$$

where $\kappa=\phi_{\star} \ln 10 / 2.5$. In Table 2 we summarize the relevant LFs (in 0.5-mag bins): the raw counts for the Coma program fields, the area-scaled counts for the control field, and the final control-field subtracted completeness-corrected galaxy LF. The completeness function we used is that given by (4) in Section 2.2. The error estimate for the final galaxy LF is derived from the Poisson uncertainties for both the control-field and program-field counts, added in quadrature.

In Figure 8 we plot this LF over over the magnitude range $15.5 \leq R \leq 22.5$ mag, the range of interest for the faint-end slope analysis (i.e., $R \simeq 15.5$ mag marks approximately 
the onset of $\mathrm{dE}$ galaxies). In the faintest three bins (i.e., for $R \gtrsim 21 \mathrm{mag}$ ) the dwarf galaxy LF appears to level off, an effect which cannot be attributed to magnitude incompleteness. These three bins are, however, relatively uncertain, as they result from a relatively large background component. By themselves, therefore, they do not provide compelling evidence for a leveling-off in the dE galaxy LF. However, Lobo et al. (1997) find evidence for a variation in the faint-end slope of the galaxy LF (steeper away from NGC 4874 and NGC 4889), and in Section 5, we describe further evidence for a paucity of faint dE galaxies in the cluster core, beginning near $R=19$ mag. Thus these features of the LF may be a manifestation of tidal disruption (or inhibited formation) of faint dEs in the dense cluster core.

The faint-end slope of our galaxy LF was obtained via weighted least-squares regression over the magnitude range $15.5 \leq R \leq 22.5 \mathrm{mag}$. The slope of the regression line is given by $\Delta \log (N) / \Delta m=0.166 \pm 0.019$; this best-fit line and its uncertainties are plotted over the corresponding magnitude range on Figure 8. This slope corresponds to $\alpha=-1.41 \pm 0.05$, and it is this value which we adopt here for the faint-end slope. (This is the value that was used in Secker \& Harris 1996 for their modeling and analysis of the composite galaxy LF.) We also analyzed a magnitude-restricted subset of our galaxy LF (the region over which our LF rises with the steepest slope, $17 \leq R \leq 21.0 \mathrm{mag}$ ), for which the resulting least-squares regression line (hatched line in Figure 8 ) yields $\alpha=-1.49 \pm 0.07$. Our adopted value for the faint-end slope of the Coma core LF is entirely consistent with the slopes obtained by Thompson \& Gregory (1993) and Bernstein et al. (1995), and it is consistent with the average of the core faint-end slopes $(\alpha=-1.55 \pm 0.12$ for the NGC 4874 and NGC 4889 regions) measured by Lobo et al. (1997). However, these measured faint-end slopes for Coma all differ significantly from the much steeper values measured for other clusters by De Propris et al. (1995), Driver et al. (1994) and Wilson et al. (1997), even though all of these LF measurements were made in the cluster cores, where we may expect the shallowest faint-end slope (Lobo et al. 1997). In addition, the seven clusters studied by De Propris et al. (1995), Driver et al. (1994) and Wilson et al. (1997) vary in Bautz-Morgan class (I - III), span a full range in richness (richness-class 0 up to 5), and all but one (A1689) has a dominant $\mathrm{cD}$ galaxy. Further deep imaging studies of rich galaxy clusters are essential to map all of the cluster parameter space and pinpoint the origin of these steep cluster luminosity functions.

In Figure 9, we plot the net Coma galaxy LF from Table 2 (solid circles) over the complete range $12 \leq R \leq 22.5$ mag. We refer to this as the composite LF, since it includes contributions from both the cluster giants and dwarfs. The contribution of the giants ( $R \lesssim 16 \mathrm{mag}$ ) to this composite LF is immediately obvious (within the scatter of small-number statistics) as a log-normal distribution peaked near $R=14.5$ mag. (Note 
that the total $R$-band magnitudes we derive for cluster giants may be underestimated; see Section 2.3 and Paper I.) In Secker \& Harris (1996) we modeled this composite LF as a sum of a Gaussian function for the giants and a Schechter function for the dEs (cf. Thompson \& Gregory 1993; Biviano et al. 1995). A least-squares fit of this Gaussian plus Schechter model for several values of the Gaussian dispersion (in each case with the faint-end slope fixed at $\alpha=-1.41 \pm 0.05)$ yielded values for the characteristic magnitude $R^{\star}$, the peak of the Gaussian distribution, and for the relative normalization factors; refer to Secker \& Harris (1996) for a full table of model parameters. The range of Gaussian dispersions which we considered to model the giant galaxies is $\sigma=0.8-1.2$ mag, constrained by the literature. In Figure 9, the solid line illustrates the best model fit $(\sigma=0.8 \mathrm{mag}$ and $\alpha=-1.41$ ), and the dotted line illustrates the corresponding decomposition of this model

fit into the two separate components. These are formally the best-fit parameters, although models with higher $\sigma(1.0-1.2 \mathrm{mag})$ also provide adequate fits.

\section{Galaxy Spatial Distributions}

The Coma cluster core is dominated in luminosity by two supergiants, the elliptical NGC $4889\left(B_{T}=12.53 \pm 0.11,(B-V)=1.04 \pm 0.01\right)$ and the cD NGC 4874 $\left(B_{T}=12.63 \pm 0.11,(B-V)=0.95 \pm 0.02\right)$ (de Vaucouleurs et al. 1991). Like many Abell clusters, Coma has significant substructure (Fitchett \& Webster 1987; Escalera, Slezak \& Mazure 1992; Davis \& Mushotzky 1993; Mohr et al. 1993; White, Briel \& Henry 1993; Colless \& Dunn 1996). In terms of optical morphology, Coma is a Rood \& Sastry (1971) B-type (binary) with NGC 4889 and NGC 4874 both obvious centers of galaxy concentration in the core. However, NGC 4874 appears special for two reasons. First, it is located at the peak of the diffuse X-ray emission in the cluster core (White, Briel \& Henry 1993) and it has a strong nuclear radio source characteristic of many central giant ellipticals (Harris 1987). Second, while both NGC 4874 and NGC 4889 have an associated globular cluster system (GCS), only NGC 4874 has the high (M87-like) specific-frequency GCS which to date has been found only in cD-like, centrally-dominant ellipticals (Harris 1987; Harris, Pritchet \& McClure 1995). For these reasons we adopt the position of NGC 4874 as the cluster center.

The distribution of giant galaxies is well described by the morphology-density (or the morphology-clustercenter radius) relationship: the morphological fraction of galaxies at any location in a galaxy cluster is independent of the cluster's global parameters (e.g., richness), and is governed primarily by the local projected galaxy density (Dressler 1980) (or the clustercentric radius; Whitmore, Gilmore \& Jones 1993). The morphology-density 
relationship also holds true for dwarf galaxies, for both the Virgo cluster (Binggeli, Tammann \& Sandage 1987; Binggeli, Tarenghi \& Sandage 1990; Ferguson \& Binggeli 1994) and the Coma cluster (Thompson \& Gregory 1993). These studies found that the early-type $\mathrm{dE}$ galaxies compose the largest number fraction of galaxies in the dense environment of the cluster core. For Coma dEs, Thompson \& Gregory (1993) determined that brighter dEs follow the distribution of the early-type giant galaxies throughout the cluster, rising steeply towards the center. They found that while the faint dEs are distributed in the same manner as the bright dEs in the outer regions, a deficit of faint $\mathrm{dE}$ galaxies was detectable within 0.35 arcmin of cluster center. Our goal is to use our large sample of program-field objects and our estimate of the background number density of galaxies to analyze the $\mathrm{dE}$ galaxy radial number-density distribution, and compare this to the radial profile for the early-type giants sharing the cluster core.

In Figure 10(a) we plot positions and magnitudes for 280 bright cluster galaxies, a subsample selected to have $0.7 \leq(B-R) \leq 1.9 \mathrm{mag}$, and $R \leq 19.0 \mathrm{mag}$ (giants plus bright dEs). Immediately noticeable is the strong clustering of bright galaxies around NGC 4874. In Figure 10(b) we plot the subsample of 2246 faint objects selected by color to $0.7 \leq(B-R) \leq 1.9 \mathrm{mag}$, and limited in magnitude to $19.0<R \geq 22.5 \mathrm{mag}$. While this sample includes the faint $\mathrm{dE}$ galaxies, it is dominated in numbers by the spatially-uniform background population. In contrast to the giants and bright dEs in (a), there is no immediately apparent fall off in the density of faint dEs, the first indication of a significantly larger core radius. Note that our sample is incomplete within a radius of 150 pixels around both NGC 4874 and NGC 4889, and these regions are excluded in the following analysis.

The radial number density profiles for the two samples of galaxies were derived by (i) summing the completeness-corrected number counts within radial annuli, (ii) normalizing these counts to the usable area of the annuli, and (iii) correcting for the number density of background objects calculated in the relevant color and magnitude range on the CONTROL field. In Figure 10 the concentric circles (dotted lines) designate the boundaries of the radial annuli used to compute the radial profile for both galaxy samples. The inner annulus begins 1.325 arcmin from NGC 4874, the outer annulus ends at $23.325 \mathrm{arcmin}$, and the region in between is divided evenly into 2 arcmin bins (except for the outer annulus; see Table [3). These are the same radial bins used for the analysis of the radial color distribution in Secker (1996). Concerning the corrections for spatial incompleteness, the actual area of the annuli used in calculations is that which intersects useable regions of the CCD images, as designated by the solid line boundary in Figure 10. Also plotted in Figure 10 (a) and (b) are incomplete regions due to NGC 4787, NGC 4889, and a very bright star north of NGC 4874. In Figure 10 (b) the small open circles are located at the positions of extended giant galaxies, and represent a first-order correction to the resultant spatial incompleteness. All 
incomplete regions are taken into account when computing the area of the annuli.

The spatially varying magnitude completeness corrections required additional simulations. The completeness function, $f(m)$, of Section 2 is a global average, derived from a sample of objects over a large and variable region of the cluster core. It was appropriate to use $f(m)$ to correct the galaxy luminosity function, since the galaxy LF represents a sum of all objects detected over these same variable regions (Section 4). However, near NGC 4874, the radial number density profile is affected by a changing object detection level: faint non-stellar objects are more likely to be missed due to the higher Poisson noise. To quantify this effect we performed artificial-star tests, and derived a separate magnitude completeness function for each of the four inner radial annuli. For the outer 6 annuli, we adopted the completeness function calculated for the fourth annulus. (At these larger radial distances, light gradients from NGC 4874 no longer affect the detection completeness, which is predominately due to sky noise.) For these simulations we used stellar profile objects, since at these faint magnitudes, the majority of the objects (stars and faint galaxies alike) have profiles close to stellar. We added a total of 1800 stars distributed as a power-law over the magnitude range $16<R<23 \mathrm{mag}$, and used a uniform spatial distribution. These stars were added to the NGC 4874 field $R$-band master image in six batches of 300 stars each; in total, 209, 388, 434 and 719 stars were added into the innermost, second, third and fourth annuli.

The resulting completeness functions for the four annuli, $f(R)$, are illustrated in Figure 11. Here, the solid circles represent the completeness fraction, and the associated uncertainties were derived using Bolte's (1989) formula, given in Section 2 as equation (3). The function $f(R)$ was computed using the number input in each 0.5-mag bin, and the number of these objects detected at any magnitude. (That is, we do not care about the effects of bin jumping, as we are only concerned with detection completeness.) For the innermost annulus, the detection completeness is $f(R)=0.80$ at our $R=22.5$ mag limit. The other three annuli are only slightly different. To these solid circles we chose to fit an analytic function, to smooth out the effects of small-number statistics, and to provide a convenient interpolation between the data points. We adopted a two-parameter function due to C.J. Pritchet, for which $\alpha$ describes the shape of the detection completeness curve, and for which $m_{\ell}$ is an effective (i.e., 50 percent complete) limiting magnitude:

$$
f(R)=\frac{1}{2}\left[1-\frac{\alpha\left(R-m_{\ell}\right)}{\sqrt{1+\alpha^{2}\left(R-m_{\ell}\right)^{2}}}\right] .
$$

With the parameters illustrated in the four panels of Figure 11, it provides a good fit (dotted line) to these completeness functions. We then used the detection completeness 
functions derived from these artificial star tests to correct the number counts in each annulus in each magnitude bin.

Finally, the completeness-corrected radial number density distributions are illustrated in Figure 12, in a plot of $\log \left(N / \operatorname{arcmin}^{2}\right)$ versus $\log \left(R_{\mathrm{cc}} / \operatorname{arcmin}\right)$. The number densities are plotted at the geometric mean radius (i.e., $r=\sqrt{r_{i} r_{j}}$ ) of the annulus, and are corrected for the mean ConTroL-field number density of objects. Within the color range $0.7 \leq(B-R) \leq 1.9 \mathrm{mag}$ and for $R \leq 19.0 \mathrm{mag}$, the control-field number density is $0.070 \pm 0.016$ objects per arcmin ${ }^{2}$. Within the same color range, and for $19.0<R \leq 22.5$ mag, the control-field number density is $2.490 \pm 0.096$ objects per $\operatorname{arcmin}^{2}$. It is clear in the upper panel of Figure 12 that the number density profile of the early-type giants and bright dEs continues to rise into our innermost bin at $R_{\mathrm{cc}}=2.1$ arcmin, while the density profile for the faint $\mathrm{dE}$ galaxies (lower panel) is very flat throughout this radial range. It is the relative differences of the core radii for these two samples of galaxies which we are primarily interested in, and below we quantify the shapes of these radial profiles using model fits to the data. Note first, however, that the outermost three points in both radial profiles correspond to only a small fraction of the total cluster area at that radius, and are thus very spatially incomplete around the annulus.

To both of these projected radial surface density profiles we fit an isotropic, single mass King model (King 1966). While these King models were originally proposed to model globular star clusters, they have also been used successfully to model galaxy clusters (Bahcall 1977; Binggelli et al. 1987). Our main interest in these models is that they fit well to our radial density profiles, and thus provide a convenient method to compare the core radius and central surface densities between our two galaxy samples. In practice, we numerically compute King models for various values of the concentration index, which we then shift vertically and horizontally until they best fit the observed data (McLaughlin et al. 1995). The model which, when shifted, provides the minimum $\chi^{2}$ statistic, is taken to be the best fit to the data. Note that the concentration index $c$ is based upon the tidal radius $r_{t}$, the estimation of which requires a large extrapolation to our radially-limited data set. Thus the values for $c$ quoted below are less certain than the estimated central density and core radius.

For the bright galaxy sample, the best-fit King model (with $\chi_{\nu}^{2}=1.43$ ) has a central surface density $\Sigma_{0}=0.53 \operatorname{arcmin}^{-2}$, a concentration index of $c=0.28$, and a core radius $R_{\mathrm{c}}=13.71$ arcmin, corresponding to $R_{\mathrm{c}}=287.1 h^{-1} \mathrm{kpc}$. Our measured core radius is larger than the value of 9 arcmin obtained by Kent \& Gunn (1982), with the difference most probably arising from our limited radial coverage. For the faint dE galaxies, the best-fit King model (with $\chi_{\nu}^{2}=1.37$ ) yields a central surface density $\Sigma_{0}=1.17 \operatorname{arcmin}^{-2}$, 
a concentration index of $c=0.28$, and a core radius $R_{\mathrm{c}}=22.15$ arcmin, corresponding to $R_{\mathrm{c}}=463.8 h^{-1} \mathrm{kpc}$. These best-fit King models are plotted along with the data points in Figure 12. There is a significant difference between these fits, in the sense that a least-squares fit of the bright-sample model to the faint-sample data (or vice versa) yields an extremely large value for the reduced chi-squared parameter.

As mentioned above, Thompson \& Gregory (1993) tentatively proposed that the core of the Coma cluster is deficient in the number of faint $\mathrm{dE}$ galaxies, and they argued that dynamical effects (tidal disruption) in the rich environment of the cluster core could be responsible for partially destroying this population of faint $\mathrm{dE}$ galaxies. However, they cautioned that this relatively flat distribution for the faint $\mathrm{dE}$ galaxies might simply be a manifestation of a uniformly distributed population of faint noncluster galaxies. Our study indicates that this flat inner distribution is genuine. Scaling the radial profile of the bright sample of galaxies to that of the faint $\mathrm{dE}$ population only, we see that the number density of the bright galaxies continues to rise farther into the core of the cluster (i.e., it has a smaller core radius), so in this sense the cluster core is indeed deficient in the numbers of faint dE galaxies. This is consistent with the results of Lobo et al. (1997), who determine that the faint-end slope of the galaxy luminosity function is shallower in the Coma cluster core, when compared to the cluster as a whole.

A further note is warranted here with respect to the destruction of dEs in the cluster core. Moore, Lake \& Katz (1997) (following from Moore et al. 1996) describe the formation of $\mathrm{dE}$ galaxies in dense clusters as morphological transformations due to gravitational interactions ("galaxy harassment"). They suggest that the radial color gradient detected in our sample of bright Coma cluster dE galaxies (Secker 1996) is a result of the destruction of faint blue dEs in the core. They believe that the absence of these faint blue dEs skews the mean galaxy color, resulting in a mean color which is redder towards the cluster center. We think that their argument is not likely to be correct for the following reasons: (a) we find that the sample of bright $\mathrm{dE}$ galaxies ( $R \leq 19.0 \mathrm{mag}$ ) has the same radial distribution as the giant galaxies, suggesting that no significant tidal disruption has occurred for them. It is only the faint $\mathrm{dE}$ galaxies (i.e., with $19<R \leq 22.5 \mathrm{mag}$ ) which have a significantly different radial number density distribution, a probable consequence of tidal disruption. (b) The color gradient noted by Secker (1996) exists over the full radial range, and it is not limited to the inner $100 \mathrm{kpc}$, as suggested by Moore et al. (1997). Thus, while we agree that the paucity of faint, fragile, blue dEs would produce a radial color gradient in the inner $100 \mathrm{kpc}$, the color gradient measured by Secker (1996) for the bright dEs can not be attributed solely to tidal disruption, and it likely represents a variation in the mean $\mathrm{dE}$ metallicity with clustercentric radius. 
One final comment concerns the effect of the different spatial distributions between luminosity-selected samples on the computation of the early-type dwarf-to-giant ratio (EDGR). Secker \& Harris (1996) used the color-restricted sample defined in Section 2.3 to estimate the EDGR for the Coma cluster. To a limiting magnitude of $R=18.6 \mathrm{mag}$, they derived a value of $1.80 \pm 0.58$, consistent with a value computed by Thompson \& Gregory (1993), and consistent with values for the Virgo cluster. The EDGR computed by Secker \& Harris (1996) is based on observations of the cluster core only, and one may question whether this is representative of the Coma cluster as a whole. Ferguson \& Sandage (1991) find no evidence to support a significant difference in their computed EDGR with a variation in clustercentric radius. They determined this by computing EDGR values in annuli of width one degree as a function of radius outwards from the Virgo cluster center. As such, they conclude that only a single value for the EDGR is necessary to characterize the Virgo cluster, and postulate that this is true for all individual galaxy clusters. To first order, this is true in the Coma cluster as well, but given the difference in spatial distributions between the cluster giants and the faint dEs determined above, this point is worth further consideration.

For large radial distance from the cluster center, Thompson \& Gregory (1993) find that at about 20 arcmin from the center (NGC 4874), the powerlaw falloff of the faint $\mathrm{dE}$ galaxies is consistent with that of the bright $\mathrm{dEs}$ and the E+S0 galaxies. Since the number of faint $\mathrm{dE}$ galaxies decreases in proportion to the number of bright galaxies, the EDGR computed at any limiting magnitude should be constant at any radius. However, this is not true for the innermost regions of the core, where the number density of bright $\mathrm{dE}$ and $\mathrm{E}+\mathrm{S} 0$ galaxies increases more rapidly than does the number density of faint $\mathrm{dE}$ galaxies. Thus a calculation for the EDGR limited to the central core of the Coma cluster, as our value is, may underestimate the EDGR for the galaxy cluster as a whole. However, this underestimation will only occur if the dE galaxy limiting magnitude (to which the Schechter function is numericallythe integrated) includes the faintest $\mathrm{dE}$ galaxies. In Secker \& Harris (1996), the first two values for the EDGR in the Coma cluster core, i.e., to limits of $R=18.6$ and $19.6 \mathrm{mag}$, are therefore representative of the Coma cluster as a whole. However, their limits of $R=20.6$ and $21.6 \mathrm{mag}$ are sufficiently faint that they include the more smoothly distributed faint $\mathrm{dE}$ galaxies, and these estimates of the EDGR value may not be applicable to the cluster as a whole. 


\section{Projected (Bound) Companions to Early-type Giants}

In the previous section we analyzed the radial number density distribution of $\mathrm{dE}$ galaxies within the cluster core, which depended only on number counts in radial annuli and magnitude bins. In this section we use the dE galaxy locations to estimate the number of dwarf galaxies which are gravitationally bound companions of early-type giants. To do this we use number counts of $\mathrm{dE}$ candidates projected near a cluster giant and subtract the local mean $\mathrm{dE}$ number density. Dwarf elliptical galaxies which formed along with the parent galaxy should be useful tracers of the extent of the giant's dark halo, as both the dEs and dark halos are affected by tidal stripping (Vader \& Chaboyer 1992; Binggeli 1993). Within the Coma cluster, there are at least two factors which come into play: (i) the more massive and luminous Es should be better able to hold onto their dwarf companions, and (ii) the dense environment of the cluster core creates extreme tidal forces. This may explain the high fraction of liberated galaxies, those moving freely in the general potential well of the cluster. However small, the number of remaining bound companions can help us to understand the evolutionary state of the cluster (Binggeli 1993). In a study of the small-scale clustering properties of dwarf galaxies, Vader and Sandage (1991) examined dwarf galaxies in the vicinity of E and E/S0 type galaxies, and determined that dwarf galaxy companions are gravitationally bound to distances of $150 \mathrm{kpc}$. Ferguson (1992) analyzed the galaxy distribution in the Virgo cluster, and estimated that on the order of 7 percent of the galaxy sample are bound companions, located within $80-150 \mathrm{kpc}$ of the primary. It is this population of dwarf companions bound to giants that we attempt to detect in Coma. Since giants in our region of the Coma cluster core are typically separated (in projection) by $\lesssim 3 \operatorname{arcmin}\left(\lesssim 63 h^{-1} \mathrm{kpc}\right)$, we avoid sample contamination due to

crowding by restricting our analysis of $\mathrm{dE}$ companions to small circular regions $\left(r \simeq 13.9 h^{-1}\right.$ $\mathrm{kpc})$ centered on the giants.

As discussed in Section 2.2 and in Secker \& Harris (1996), our dE galaxy detections are incomplete very near the cluster giants. To alleviate this problem, we modeled and subtracted the intensity profiles of 10 giants, detected and measured anew all objects in these regions, and then matched object lists to yield a consistent and complete catalog for these regions. The isophotal models were constructed within IRAF using the tasks ellipse and bmodel of the isophote package within STSDAS. These tasks implement the method of Jedrzejewski (1987) for iterative modeling of elliptical isophotes, and allow for variable center position, ellipticity $\epsilon$ and position angle $\theta$ at each semimajor axis radius $r$. In all cases, we allowed $\epsilon$ and $\theta$ to vary freely, in order to achieve the best subtraction of the galaxy light (Figure 13). In all cases the subtraction was excellent over the entire radial range, with the exception of the centermost pixels. We cross-referenced our positions for these ten galaxies with the compilation of Kent \& Gunn (1982): Table 4 provides the 
NGC/IC names, $(\alpha, \delta)$ and pixel positions, total $R$ magnitudes ( $2 r_{1}$ aperture magnitudes), and the radial extent of the models for the 10 giants considered here.

With the light profiles of the giants subtracted, we proceeded to detect objects in these areas, using the same analysis methods described in Paper I: the galaxy-subtracted $R$ and $B$-band master images were ring median filtered, after which our DYNAMO software (Paper I) was used to detect and measure objects. Total $R$ magnitudes and $(B-R)$ colors were used to select a color- and magnitude-restricted subsample of objects (as described in Section 2.4), and objects falling on the bright-stellar sequence were culled from the list. The new object lists for the areas around the 10 giant galaxies was then merged with the final object sample described in Section 2.3, eliminating any objects which are common to both samples. Near the center positions of the ten subtracted galaxies, the Poisson noise inherent in the image is the greatest. Because of the problem that this introduces for detection completeness, and because of poor galaxy subtraction, these small circular regions were excluded from the analysis. In addition to these central regions, three of the ten giants were sufficiently close together that the areas of consideration around them overlapped; for these cases, wedge-shaped areas which included the adjacent galaxies were omitted from the analysis. In all cases the areas used to compute number densities were corrected for these excluded areas.

Using this spatially complete subsample of objects, we performed number counts of objects in circular regions of radius 75 pixels centered on each giant; dividing by the total useable area yielded the corresponding number densities, $\sigma_{\text {gal }}$. With $N$ and $A$ representing the number of detections and the effective area, we have $\sigma_{\text {gal }}=N / A \pm \sqrt{N} / A$. For statistical background correction, we computed a local background number density, $\sigma_{\mathrm{bkg}}$, near the position of each giant. By correcting with $\sigma_{\mathrm{bkg}}$, we forgo the need to deproject the 2D galaxy distribution to a 3D one, since on a statistical basis, any excess of galaxies above $\sigma_{\mathrm{bkg}}$ will be a result of companion galaxies. The resulting number densities are provided in Table 4 . The calculation for the background-subtracted number of objects projected within the 75 pixel radius, $N_{75, i}$, around each giant galaxy, is given by

$$
N_{75, i}=A_{i}\left(\sigma_{\mathrm{gal}, i}-\sigma_{\mathrm{bkg}, i}\right)
$$

Values of $N_{75, i}$ for our sample of 10 giants are given in Table 4 . On average, there are $4 \pm 1$ objects per giant in excess of the local background levels, indicating that we have evidence for a small but significant population of bound companions. While this result is consistent with the vast majority of dEs being unbound and free to move in the global cluster potential, it also suggests that there is a measurable population of bound companions around the most luminous giants in the cluster core. A more sophisticated follow-up analysis would 
be warranted, to study a wider range of giant galaxies, with a greater range of position in the cluster and total magnitude. In Figure 14 we plot $N_{75}$ versus $R$ magnitude for each of the ten giant galaxies. While there is significant scatter, there is a weak trend of increasing density with increasing luminosity of the giant galaxy. A weighted least-squares fit yields a best-fit line with a slope of $\Delta N_{75} / \Delta R=-5.2 \pm 2.9$. While this result is only of slight statistical significance, it works in the sense that the more luminous and therefore massive galaxies are better able to bind their companion galaxies, as expected.

This paper is based upon thesis research conducted by J.S. while at McMaster University. The research was supported in part by: the Natural Sciences and Engineering Research Council of Canada (through a grant to W.E.H.), the Department of Physics and Astronomy at McMaster University, a grant to J.S. from NASA administered by the American Astronomical Society, a Fullam Award to J.S. from Dudley Observatory, and the Ontario Ministry of Colleges and Universities (through an Ontario Graduate Scholarship to J.S). We would like to thank P.R. Durrell, D.E. McLaughlin, C.J. Pritchet and the referee, J. Loveday, for their helpful comments, and to thank S. Holland for his help with the observations. 
Table 1. Calibration summary for April 1991 KPNO 4m run.

\begin{tabular}{ccccccccc}
\hline \hline NIGHT & $\begin{array}{c}\text { Total } \\
\text { Standards }\end{array}$ & Filter & $Z P_{R}$ & $a_{1}$ & $a_{2}$ & $Z P_{B}$ & $a_{3}$ & $a_{4}$ \\
\hline 1 & 71 & $\mathrm{R}$ & $22.418 \pm 0.016$ & 0.130 & $-0.002 \pm 0.013$ & - & - & - \\
1 & 59 & $\mathrm{~B}$ & - & - & - & $21.081 \pm 0.016$ & 0.283 & $-0.042 \pm 0.014$ \\
2 & 46 & $\mathrm{R}$ & $22.408 \pm 0.018$ & 0.130 & $-0.002 \pm 0.013$ & - & - & - \\
2 & 34 & $\mathrm{~B}$ & - & - & - & $21.109 \pm 0.018$ & 0.283 & $-0.042 \pm 0.014$ \\
\hline
\end{tabular}

${ }^{1}$ Observations made with the TE $2 \mathrm{~K}$ CCD at PF of the KPNO 4m on April 9th and 10th, 1991.

${ }^{2}$ No errors are provided for $a_{1}$ or $a_{3}$, average CTIO extinction coefficients from Landolt (1983). 
Table 2. Luminosity Function For Coma Cluster Galaxies

\begin{tabular}{rrrrrrrrrr}
\hline \hline$R$ mag & $N_{\text {Galaxy }}$ & $N_{\text {Background }}$ & $f(R)$ & $N_{\text {Total }}$ & $R$ mag & $N_{\text {Galaxy }}$ & $N_{\text {Background }}$ & $f(R)$ & $N_{\text {Total }}$ \\
\hline 12.75 & 1 & - & 1.000 & $1 \pm 1$ & 17.75 & 30 & 2.6 & 1.000 & $27 \pm 6$ \\
13.25 & 6 & - & 1.000 & $6 \pm 2$ & 18.25 & 49 & 2.6 & 0.989 & $47 \pm 7$ \\
13.75 & 10 & - & 1.000 & $10 \pm 3$ & 18.75 & 61 & 28.3 & 0.967 & $34 \pm 10$ \\
14.25 & 16 & 2.6 & 1.000 & $13 \pm 4$ & 19.25 & 94 & 48.9 & 0.944 & $48 \pm 12$ \\
14.75 & 13 & - & 1.000 & $13 \pm 4$ & 19.75 & 135 & 61.8 & 0.922 & $79 \pm 15$ \\
15.25 & 22 & - & 1.000 & $22 \pm 5$ & 20.25 & 202 & 131.4 & 0.900 & $79 \pm 19$ \\
15.75 & 9 & - & 1.000 & $9 \pm 3$ & 20.75 & 267 & 164.9 & 0.878 & $116 \pm 22$ \\
16.25 & 17 & 2.6 & 1.000 & $14 \pm 4$ & 21.25 & 346 & 293.7 & 0.856 & $61 \pm 27$ \\
16.75 & 23 & 5.2 & 1.000 & $18 \pm 5$ & 21.75 & 481 & 386.4 & 0.833 & $114 \pm 32$ \\
17.25 & 23 & 5.2 & 1.000 & $18 \pm 5$ & 22.25 & 721 & 651.7 & 0.811 & $85 \pm 41$ \\
\hline
\end{tabular}

${ }^{1}$ The number counts in column 3 includes a correction for the ratio of areas, $A_{p} / A_{c}=2.576$.

${ }^{2} N_{\text {Total }}=I N T\left[f \times\left(N_{\text {Galaxy }}-N_{\text {Background }}\right)\right]$. 
Table 3. Radial Number Densities For Cluster Galaxies

\begin{tabular}{rrrrrrrrr}
\hline \hline \multicolumn{1}{c}{$R_{\text {in }}^{\prime}$} & $R_{\text {out }}^{\prime}$ & $\bar{R}^{\prime}$ & $N_{\text {bright }}$ & $\begin{array}{c}\text { Area } \\
\operatorname{arcmin}^{2}\end{array}$ & $\begin{array}{c}\sigma_{\text {bright }} \\
N / \text { arcmin }^{2}\end{array}$ & $N_{\text {faint }}$ & $\begin{array}{c}\text { Area } \\
\operatorname{arcmin}^{2}\end{array}$ & $\begin{array}{c}\sigma_{\text {faint }} \\
N / \text { arcmin }^{2}\end{array}$ \\
\hline 1.33 & 3.33 & 2.10 & $25 \pm 5.0$ & 29.28 & $0.78 \pm 0.17$ & $101 \pm 10$ & 28.04 & $1.11 \pm 0.39$ \\
3.33 & 5.33 & 4.21 & $29 \pm 5.4$ & 53.91 & $0.47 \pm 0.10$ & $205 \pm 15$ & 52.77 & $1.40 \pm 0.31$ \\
5.33 & 7.33 & 6.25 & $34 \pm 5.8$ & 76.96 & $0.39 \pm 0.08$ & $236 \pm 16$ & 73.01 & $0.74 \pm 0.24$ \\
7.33 & 9.33 & 8.27 & $48 \pm 6.9$ & 102.60 & $0.41 \pm 0.07$ & $365 \pm 20$ & 99.20 & $1.19 \pm 0.22$ \\
9.33 & 11.33 & 10.28 & $40 \pm 6.3$ & 93.03 & $0.36 \pm 0.07$ & $312 \pm 18$ & 92.50 & $0.88 \pm 0.22$ \\
11.33 & 13.33 & 12.29 & $29 \pm 5.4$ & 77.25 & $0.31 \pm 0.07$ & $300 \pm 18$ & 76.34 & $1.44 \pm 0.26$ \\
13.33 & 15.33 & 14.29 & $33 \pm 5.7$ & 70.08 & $0.40 \pm 0.08$ & $213 \pm 15$ & 68.82 & $0.61 \pm 0.24$ \\
15.33 & 17.33 & 16.30 & $13 \pm 3.6$ & 68.83 & $0.12 \pm 0.05$ & $236 \pm 16$ & 68.77 & $0.94 \pm 0.25$ \\
17.33 & 19.33 & 18.30 & $13 \pm 3.6$ & 58.35 & $0.15 \pm 0.06$ & $189 \pm 14$ & 57.73 & $0.78 \pm 0.27$ \\
19.33 & 23.33 & 21.23 & $16 \pm 4.0$ & 61.66 & $0.19 \pm 0.07$ & $181 \pm 14$ & 61.53 & $0.45 \pm 0.25$ \\
\hline
\end{tabular}

${ }^{1}$ The bright sample includes 280 objects brighter than $R=19 \mathrm{mag}$, while the faint sample includes $2246 \mathrm{dE}$ galaxies with $19.0<R \leq 22.5 \mathrm{mag}$. Both samples are incomplete within 150 pixels (1.325 arcmin) of NGC 4874 and NGC 4889. The number counts given in columns 4 and 7 are corrected for magnitude incompleteness.

${ }^{2}$ The slight differences in annular area between the bright and faint samples results from the excluded regions illustrated in Figure 10(b).

${ }^{3}$ The number densities given in columns 6 and 9 are corrected for the mean control field levels: $0.23 \pm 0.03$ per $\operatorname{arcmin}^{2}$ and $2.33 \pm 0.09$ per $\operatorname{arcmin}^{2}$ respectively.

${ }^{4} \bar{R}=\sqrt{R_{\text {in }} R_{\text {out }}}$, the geometric mean radius. 
Table 4. Background-Corrected Number Counts Centered on Early-type Cluster Giants

\begin{tabular}{lccccccccc}
\hline \hline NGC/IC & $\begin{array}{c}\alpha \\
(2000)\end{array}$ & $\begin{array}{c}\delta \\
(2000)\end{array}$ & $\begin{array}{c}X \\
\mathrm{px}\end{array}$ & \multicolumn{1}{c}{$\begin{array}{c}\mathrm{p} \text { px } \\
\text { N }\end{array}$} & $\begin{array}{c}R_{\text {bmodel }} \\
\mathrm{px}\end{array}$ & $\begin{array}{c}R \\
\mathrm{mag}\end{array}$ & $\begin{array}{c}\sigma_{\text {bkg }} \\
N / \operatorname{arcmin}^{2}\end{array}$ & $\begin{array}{c}\sigma_{\text {gal }} \\
N / \operatorname{arcmin}^{2}\end{array}$ & $N$ \\
\hline IC 3957 & $12: 59: 06.9$ & $27: 46: 13.6$ & 342.84 & -288.79 & 58.3 & 14.26 & $3.5 \pm 0.5$ & $3.4 \pm 3.4$ & $4.7 \pm 4.6$ \\
IC 3959 & $12: 59: 08.1$ & $27: 47: 13.6$ & 361.75 & -177.71 & 68.9 & 13.73 & $3.5 \pm 0.5$ & $3.4 \pm 3.4$ & $4.7 \pm 4.6$ \\
IC 3963 & $12: 59: 12.9$ & $27: 46: 37.7$ & 493.90 & -243.61 & 50.4 & 14.23 & $3.5 \pm 0.5$ & $5.5 \pm 3.0$ & $7.5 \pm 4.1$ \\
IC 3960 & $12: 59: 08.0$ & $27: 51: 25.6$ & 358.10 & 305.42 & 39.8 & 14.22 & $4.4 \pm 0.9$ & $1.7 \pm 2.5$ & $2.4 \pm 3.4$ \\
NGC 4883 & $12: 59: 55.9$ & $28: 02: 02.5$ & 1572.88 & 1519.05 & 37.1 & 13.85 & $3.6 \pm 0.7$ & $-0.7 \pm 1.5$ & $-0.9 \pm 2.1$ \\
IC 4026 & $13: 00: 21.6$ & $28: 02: 44.9$ & 2228.01 & 1598.08 & 37.1 & 14.21 & $6.0 \pm 0.5$ & $-1.6 \pm 1.9$ & $-2.1 \pm 2.6$ \\
IC 4045 & $13: 00: 48.5$ & $28: 05: 21.5$ & 2893.21 & 1892.28 & 37.1 & 13.55 & $5.3 \pm 0.5$ & $0.7 \pm 2.1$ & $0.9 \pm 2.9$ \\
NGC 4908 & $13: 00: 51.5$ & $28: 02: 27.5$ & 2963.71 & 1565.89 & 42.4 & 13.30 & $3.3 \pm 0.6$ & $8.6 \pm 3.0$ & $11.8 \pm 4.2$ \\
NGC 4906 & $13: 00: 39.6$ & $27: 55: 27.3$ & 2664.24 & 760.03 & 37.1 & 13.89 & $3.1 \pm 0.3$ & $1.4 \pm 1.8$ & $1.9 \pm 2.5$ \\
IC 4051 & $13: 00: 53.3$ & $28: 00: 21.5$ & 3035.21 & 1326.23 & 90.1 & 13.05 & $3.3 \pm 0.7$ & $5.6 \pm 2.7$ & $7.7 \pm 3.7$ \\
\hline
\end{tabular}

\footnotetext{
${ }^{1}$ The magnitudes given in column 5 were derived using $2 r_{1}$ apertures; as noted in Paper I, these underestimate the total magnitude for de Vaucouleurs-profile galaxies such as these.

${ }^{2} \sigma_{\text {gal }}$ and $N$ represent the background-corrected number density and number counts within a circle of 75 pixel radius centered on the $(X, Y)$ center of the galaxy.
} 


\section{REFERENCES}

Babul, A. \& Rees, M.J. 1992, MNRAS, 255, 346

Bahcall, N.A. 1977, ARA\&A, 15, 505

Bender, R., Paquet, A. \& Nieto, J.-L. 1991, A\&A, 246, 349

Bernstein, G.M., Nichol, R.C., Tyson, J.A., Ulmer, M.P. \& Wittman, D. 1995, AJ, 110, 1507

Binggeli, B. 1993, Habilitationsschrift, Universität Basel

Binggeli, B., Tammann, G.A. \& Sandage, A. 1987, AJ, 94, 251

Binggeli, B., Sandage, A. \& Tammann, G.A. 1988, ARA\&A, 26, 509

Binggeli, B., Tarenghi, M. \& Sandage, A. 1990, A\&A, 228, 42

Biviano, A., Durret, F., Gerbal, D., Le Fèvre, O., Lobo, C., Mazure, A. \& Slezak, E. 1995, A\&A, 297, 610

Bolte, M. 1989, ApJ, 341, 168

Caldwell, N. \& Bothun, G.D. 1987, AJ, 94, 1126

Cellone, S.A., Forte, J.C. \& Geisler, D. 1994, ApJS, 93, 397

Colless, M. \& Dunn, A.M. 1996, ApJ, 458, 435

Couture, J., Harris, W.E. \& Allwright, J.W.B. 1990, ApJS, 73, 671

Couture, J., Harris, W.E. \& Allwright, J.W.B. 1991, ApJ, 372, 97

Davis, D.S. \& Mushotzky, R.F. 1993, AJ, 105, 409

Davis, L. 1990, private communication (Globular Cluster standard star photometry, unpublished)

De Propris, R., Pritchet, C.J., Harris, W.E. \& McClure, R.D. 1995, ApJ, 450, 534

de Vaucouleurs, G., de Vaucouleurs, A., Corwin, H.G., Jr., Buta, R.J., Paturel, G. \& Fouqué, P. 1991, Third Reference Catalogue of Bright Galaxies (Springer, New York)

Dekel, A. \& Silk, J. 1986, ApJ, 303, 39

Dressler, A. 1980, ApJ, 236, 351

Driver, S.P., Phillipps, S., Davies, J.I., Morgan, I. \& Disney, M.J. 1994, MNRAS, 268, 393

Durrell, P.R., Harris, W.E. \& Pritchet, C.J. 1994, AJ, 108, 2114

Durrell, P.R. 1997, AJ, 113, 531

Escalera, E., Slezak, E. \& Mazure, A. 1992, A\&A, 264, 379 
Evans, R.H., Davies, J.I. \& Phillipps, S. 1990, MNRAS, 245, 164

Ferguson, H.C. 1992, MNRAS, 255, 389

Ferguson, H.C. \& Sandage, A. 1988, AJ, 96, 1520

Ferguson, H.C. \& Sandage, A. 1991, AJ, 101, 765 (FS91)

Ferguson, H.C. \& Binggeli, B. 1994, A\&A Rev, 6, 67-122

Fitchett, M. \& Webster, R. 1987, ApJ, 317, 653

Gallagher, J.S., III \& Wyse, R.F.G. 1994, PASP, 106, 1225-1238

Garilli, B., Bottini, D., Maccagni, D., Vettolani, G. \& Maccacaro, T. 1992, AJ, 104, 1290

Geisler, D. \& Forte, J.C. 1990, ApJ, 350, L5

Harris, W.E. 1987, ApJ, 315, L29

Harris, W.E. 1996, AJ, 112, 1487

Harris, W.E., Pritchet, C.J. \& McClure, R.D. 1995, ApJ, 441, 120

Harris, W.E., Fitzgerald, M.P., and Reed, B.C. 1981, PASP, 93, 507

Held, E. \& Mould, J.R. 1994, AJ, 107, 1307

Ichikawa, S., Wakamatsu, K. \& Okamura, S. 1986, ApJS, 60, 475

Jedrzejewski, R. I. 1987, MNRAS, 226, 747

Jerjen, H. \& Binggeli, B. 1997, in The Nature of Elliptical Galaxies, Proceedings of the Second Stromlo Symposium, Eds. M. Arnaboldi, G.S. Da Costa \& P. Saha

Kent, S.M. \& Gunn, J.E. 1982, AJ, 87, 945

King, I.R. 1966, AJ, 71, 64

Kormendy, J. 1977, ApJ, 218, 333

Kuhn, J.R., Smith, H.A. \& Hawley, S.L. 1996, ApJ, 469, L93

Landolt, A. 1983, AJ, 88, 439

Lobo, C., Biviano, A., Durret, F., Gerbal, D., Le Fèvre, O., Mazure, A. \& Slezak, E. 1997, A\&A, 317, 385

Mattila, K. 1977, A\&A, 60, 425

McLaughlin, D.E., Secker, J., Harris, W.E. \& Geisler, D. 1995, AJ, 109, 1033

Melnick, J., White, S.D.M \& Hoessel, J. 1977, MNRAS, 180, 207

Mohr, J.J., Fabricant, D.G. \& Geller, M.J. 1993, ApJ, 413, 492

Moore, B., Katz, N., Lake, G., Dressler, A., \& Oemler, A. 1996, Nature, 379, 613 
Moore, B., Lake, G. \& Katz, N. 1997, ApJ, submitted (astro-ph/9701211)

Peterson, R.C. \& Caldwell, N. 1993, AJ, 105, 1411

Press, W.H. \& Schechter, P. 1974, ApJ, 187, 425

Rood, H.J. \& Sastry, G.N. 1971, PASP, 83, 313

Sandage, A. \& Binggeli, B. 1984, AJ, 89, 919

Sandage, A., Binggeli, B. \& Tammann, G.A. 1985, AJ, 90, 1759

Sarajedini, A. \& Layden, A.C. 1995, AJ, 109, 1086

Schechter, P. 1976, ApJ, 203, 297

Schild, R.E. 1983, PASP, 95, 1021

Schombert, J.M., Pildis, R.A., Eder, J.A. \& Oemler, A.Jr. 1995, AJ, 110, 2067

Secker, J. 1996, ApJ, 469, L81

Secker, J., Geisler, D., McLaughlin, D.E. \& Harris, W.E. 1995, AJ, 109, 1019

Secker, J. \& Harris, W.E. 1996, ApJ, 469, 623

Secker, J. \& Harris, W.E. 1997, PASP, 109, in press (Paper I)

Smecker-Hane, T.A., Stetson, P.B., Hesser, J.E. \& Lehnert, M.D. 1994, AJ, 108, 507

Stetson, P.B. 1987, PASP, 99, 191

Thompson, L.A. \& Gregory, S.A. 1980, ApJ, 242, 1

Thompson, L.A. \& Gregory, S.A. 1993, AJ, 106, 2197

Thuan, T.X. \& Kormendy, J. 1977, PASP, 89, 466

Uson, J.M., Boughn, S.P. \& Kuhn, J.R. 1991, ApJ, 369, 46

Vader, J.P., \& Sandage, A. 1991, ApJ, 379, L1

Vader, J.P., \& Chaboyer, B. 1992, PASP, 104, 57

van den Bergh, S. 1986, AJ, 91, 271

White, S.D.M., Briel, U.G. \& Henry, J.P. 1993, MNRAS, 261, L8

Whitmore, B.C., Gilmore, D.M. \& Jones, C. 1993, ApJ, 407, 489

Wilson, G., Smail, I., Ellis, R.S. \& Couch, W.J. 1997, submitted to MNRAS astroph/9610199)

Worthey, G. 1994, ApJS, 95, 107 
Fig. 1. - The magnitude completeness function was derived by comparing photometry for overlapping regions of the program field. The solid circles represent the completeness function for objects restricted to the color range $0.7 \leq(B-R) \leq 1.9 \mathrm{mag}$, the color range of our dE galaxy sample. The open circles represent the completeness function for all objects, shown here for comparison. The straight dashed line is our adopted completeness function, illustrating that our final object lists are 80 percent complete at the limiting magnitude $R_{2 r_{1}}=22.5$ mag.

Fig. 2. - Total magnitude and color comparison for objects in common between the NGC 4874 field and the other two the program fields. Crosses denote the 416 objects on the NGC 4889 field, while the solid circles denote the 448 objects on the NGC 4874 SoutH field. (Upper Panel) The scatter in the magnitude estimates is consistent with photometric error, yet there is a slight $\simeq 0.05$-mag bias between total magnitudes measured on the NGC 4889 field and those measured on the NGC 4874 field. (Lower panel) The scatter in the $(B-R)$ color estimates is consistent with photometric error, and there is no evidence for bias between fields.

Fig. 3. - $512 \times 512$ pixel $^{2}$ sections of the master $R$-band CCD images, with North upwards and East to the right. On each of the images, the most luminous cluster dEs are labeled. A fraction of the fainter $\mathrm{dE}$ galaxies are obvious as centrally-concentrated low-surfacebrightness objects; however, these fainter objects also consist of foreground stars and noncluster galaxies: (a) Northeast of NGC 4889. The $R$-band total magnitudes and $(B-R)$ colors for objects 1 through 6 are: $(16.82,1.58)$; $(17.24,1.27) ;(17.84,1.43)$; $(18.49,1.47)$; $(18.53,1.38) ;(18.79,1.29)$. (b) South of NGC 4874. The $R$-band total magnitudes and $(B-R)$ colors for objects 1 through 4 are: $(17.69,1.38) ;(17.86,1.41) ;(17.94,1.41) ;(19.13$, $1.32)$.

Fig. 4.- Composite CMD For The Coma Cluster Core. (Left panel) The object lists for the three program fields have been added together, and the final composite CMD for 3723 objects (2526 within the restricted color range) is illustrated here. (Right panel) The control field CMD is replotted within the same limits, for comparison. It consists of a total of 1164 objects, 694 of which are within the restricted color range.

Fig. 5.- Variation of the $r_{1}$ radius with total $R$ magnitude. The scale radius of the cluster galaxies is traced by the radial moment $r_{1}$, and plotted here as a function of the apparent total $R$ magnitude. We have plotted the color-restricted sample of program-field objects (left panel) along with the color-restricted sample of control field objects (right panel); the cluster galaxies are apparent as an excess of nonstellar objects at all magnitudes.

Fig. 6. - The dependence of central surface brightness $I_{\mathrm{c}}$ as a function of total $R$ magnitude, 
for the color restricted sample of program-field objects (left panel) and control-field objects (right panel). The stellar sequence can be seen to begin at $R=19.5 \mathrm{mag}$, and the cluster galaxies lie in a diffuse band at lower $I_{\mathrm{c}}$.

Fig. 7.- An analysis of the color-magnitude correlation for the cluster dE galaxies. (left panel) Reproduced here is the color-restricted sample of program-field objects, shown as the small points. The solid circles represent the median color values (in 1-mag bins) of these program-field objects, while the open circles indicate the median color values (in the same 1mag bins) for the control-field objects. The solid line is a regression line fit to the five points shown; the dashed line is an extrapolation of the above line to fainter magnitudes. (right panel) The program- and control-field CMDs were binned, and an area-scaled control-field CMD was subtracted from the program-field CMD. There is one vertical line segment per color-magnitude bin, with a height proportional to the number of objects in the bin. The bins have a height of $1.0 \mathrm{mag}$, and a width of $0.1 \mathrm{mag}$. The maximum number of objects in a bin is 50, while several bins contain a negative number of objects. The solid circles correspond to the mean galaxy color (an average color weighted by the number of objects per bin) in a 1-mag bin. The regression line from the left panel provides an excellent fit over the entire magnitude range. This fiducial $\mathrm{dE}$ sequence indicates that a one magnitude brightening in $R$ corresponds to a redder color by $(B-R)=0.056$ mag.

Fig. 8.- The faint-end of the completeness-corrected and background-subtracted luminosity function for our sample of Coma cluster galaxies, as defined in Table 2. The solid line is a weighted least-squares fit over the range shown, which yields a slope of $\alpha=-1.41 \pm 0.05$ for the Schechter luminosity function.

Fig. 9.- The composite galaxy luminosity function (LF) for the Coma cluster core was decomposed into the contribution from the giants (i.e., a log-normal distribution) and from the dwarf ellipticals (i.e., a Schechter function). The models have a Gaussian dispersion which varies between $\sigma=0.8 \mathrm{mag}$ and $\sigma=1.2 \mathrm{mag}$; Three of these models are illustrated in this plot. The best-fit model has $\sigma=0.80 \mathrm{mag}$, and for this case we illustrate the decomposition of the model into its two components (dotted lines). Note that the three faintest points were not included in the fit, as they adversely affect normalization over the region of interest.

Fig. 10.- The spatial distribution of candidate cluster members. (a) Program-field objects brighter than $R=19$ mag (mostly cluster Es and dEs), show a strong clustering around NGC 4874 and NGC 4889, located in this plot at $(1061,1010)$ and $(1875,1124)$ respectively. The small dotted circles denote incomplete regions; (b) Program-field objects fainter than $R=19$ mag (faint cluster dEs and a uniformly distributed background population). The 
large dotted circles are centered on NGC 4874, and denote the annular boundaries used for computing the radial surface density profiles. The small solid circles denote incomplete areas, corresponding to NGC 4874, NGC 4889, a bright star, and several other extended galaxies.

Fig. 11. - The detection completeness functions derived from artificial star simulations. The four radial zones are numbered such that the innermost zone around NGC 4874 is Zone 1. The solid circles and associated uncertainties were computed knowing the number of artificial stars input in each magnitude bin, and from the number of these objects which were subsequently detected. The dotted lines illustrate least-squares fits of an appropriate interpolation function.

Fig. 12.- Radial number density profile $\log \left(N / \operatorname{arcmin}^{2}\right)$ versus $\log \left(R_{\mathrm{cc}} / \operatorname{arcmin}\right)$ for Coma cluster galaxies, plotted over the clustercentric range $1.33 \leq R_{\mathrm{cc}} \leq 23.33$ arcmin. The top panel correspond to the sample of cluster $\mathrm{E}$ and bright $\mathrm{dEs}$, and the bottom panel corresponds to the sample of faint dEs. while the solid line gives the best-fit King model. The solid line illustrates the best-fit King model, which in both cases provides an excellent fit to the observed distributions.

Fig. 13. - Original and model-subtracted images of five cluster giants. The upper left section of the image illustrates the cluster giants IC 3957, IC 3959 and IC 3963 (Table 4), while the upper right shows the image after isophotal modeling and subtraction. The lower left image shows NGC 4908 and IC 4051; the lower right image shows the image after these two galaxies have been subtracted. In all cases the isophotal models subtracted very well, except for small regions at the galaxy centers.

Fig. 14. - The background-subtracted number of objects within a 75 pixel radius around each of the ten giant Es in the cluster core is plotted versus an estimate of the total $R$-band magnitude of the giants. The weighted least-squares regression line is also shown, though it has low statistical significance. 